\title{
Period Variations and Binary Hypotheses of Three $\delta$ Scuti stars: CY Aqr, BS Aqr, AD CMi
}

\author{
Jian Ning Fu \\ Laboratoire d'Astrophysique, Observatoire Midi-Pyrénées, 14 avenue \\ Édouard Belin, F-31400 Toulouse, France
}

\begin{abstract}
Some new times of light maxima for three $\delta$ Scuti stars CY Aqr, BS Aqr and AD CMi - have been determined based on new photoelectric photometry. Incorporated with the times presented in the literature, the $\mathrm{O}-\mathrm{C}$ method is used to study the period variations of these stars with a newly-developed program OMC. The results reveal that for these three stars, the binary hypothesis concerning a low mass companion is reasonable in explaining the $\mathrm{O}-\mathrm{C}$ diagrams.
\end{abstract}

\section{Introduction}

Since some high-amplitude $\delta$ Scuti stars have long time-span observations, it is possible to calculate their period change rates using the $\mathrm{O}-\mathrm{C}$ method. Among them, CY Aqr, BS Aqr, and AD CMi are some of the most extensively observed $\delta$ Scuti stars since the discovery of their light variations (Hoffmeister 1931, 1934a, $1934 \mathrm{~b})$. So, these three $\delta$ Scuti stars were selected to make new observations and further $\mathrm{O}-\mathrm{C}$ studies.

\section{New Observations}

The new observations were made at the Xinglong station of Beijing Astronomical Observatory with a $60-\mathrm{cm}$ telescope plus a CCD camera. CY Aqr was observed on 21 Dec 1995 with one new time of light maximum determined: T=HJD 2450072.9916; combined with those in the literature, 488 times are presented for CY Aqr. BS Aqr was observed on 20 Dec 1995 with one new time of light maximum derived: $T=$ HJD 2450072.0441; 56 times are collected over more than six decades for BS Aqr. AD CMi was measured on 19 Dec 1995. One new time of light maximum was determined: $T=\mathrm{HJD} 2450071.2184$, and 49 data points are collected over about 36 years for $\mathrm{AD}$ CMi.

\section{The Program OMC for $\mathrm{O}-\mathrm{C}$ Calculations}

The data are analyzed with the program OMC (Fu et al. 1998). This program makes linear a least-squares fit, a quadratic fit, and a quadratic plus trigonometric function fit to the data, based on the formulae of $C_{1}=T_{01}+P_{01} E$, 
$C_{\mathrm{q}}=T_{02}+P_{02} E+\frac{1}{2} \beta_{01} E^{2}$, and $C_{\text {orb }}=T_{03}+P_{03} E+\frac{1}{2} \beta_{02} E^{2}+A \sin \phi+B \cos \phi$, respectively, providing $\mathrm{O}-\mathrm{C}$ diagrams, fitting parameters and residuals.

\section{Period Variations and Binary Hypotheses}

Table 1 lists the $\mathrm{O}-\mathrm{C}$ fitting results for $\mathrm{CY}$ Aqr, BS Aqr, and AD CMi. From the $\mathrm{O}-\mathrm{C}$ diagrams, which are not shown in present paper, and the residuals after different fits, one finds that the model of explaining the discrepancies between the observed and calculated times of maximum light as the consequence of a continuously changing period combined with the light-time effect caused by the orbital motion of CY Aqr, BS Aqr, AD CMi around the mass centers of binary system with an unseen companion, is reasonable. The rates of period change of CY Aqr and $\mathrm{AD} \mathrm{CMi,} \beta_{02}=0.21 \times 10^{-8} \mathrm{~d} / \mathrm{yr}, 0.10 \times 10^{-8} \mathrm{~d} / \mathrm{yr}$ respectively, are in agreement with the predictions of the stellar evolution theory both in the direction of the period changes (increasing) and the value of the rate of change $\left(\sim 0.10 \times 10^{-8} \mathrm{~d} / \mathrm{yr}\right)$. For BS Aqr, $\beta_{02}=-0.12 \times 10^{-8} \mathrm{~d} / \mathrm{yr}$ agrees with the expected value of rate of change, but not with the direction.

Table 1. O-C fitting results for CY Aqr, BS Aqr, and AD CMi

\begin{tabular}{cccc}
\hline & CY Aqr & BS Aqr & AD CMi \\
\hline$T_{01}$ [HJD] & 2426159.5057 & 2428095.3342 & 2436601.8209 \\
$P_{01}$ [d] & 0.061038339 & 0.197822622 & 0.122974494 \\
$\sigma_{01}$ [d] & 0.0034 & 0.0039 & 0.0019 \\
$T_{02}$ [HJD] & 2426159.5008 & 2428095.3327 & 2436601.8223 \\
$P_{02}$ [d] & 0.061038383 & 0.197822691 & 0.122974345 \\
$\beta_{01}$ [d/c] & $-8.63 \times 10^{-14}$ & $-5.54 \times 10^{-13}$ & $1.52 \times 10^{-12}$ \\
$\sigma_{02}$ [d] & 0.0032 & 0.0038 & 0.0012 \\
$T_{03}$ [HJD] & 2426159.5000 & 2428095.3320 & 2436601.8203 \\
$P_{03}$ [d] & 0.061038256 & 0.197822690 & 0.122974463 \\
$\beta_{02}$ [d/c] & $3.59 \times 10^{-13}$ & $-6.74 \times 10^{-13}$ & $3.50 \times 10^{-13}$ \\
$A$ [d] & 0.0035 & -0.0040 & 0.0012 \\
$B$ [d] & -0.0108 & 0.0005 & 0.0022 \\
$P_{\text {orb }}$ [yr] & 63.15 & 31.54 & 30.44 \\
$\mathrm{e}$ & 0.28 & 0.50 & 0.30 \\
$\sigma_{03}$ [d] & 0.0012 & 0.0040 & 0.0021 \\
\hline & & &
\end{tabular}

\section{References}

Fu, J. N., Jiang, S. Y., \& Zhou, A. Y. 1998, Publ. Beijing Astron. Obs. 32, 35 Hoffmeister, C. 1931, Astron. Nachr., 242, 128

Hoffmeister, C. 1934a, Beobachtungszirkular Astron. Nachr., 16, 45

Hoffmeister, C. 1934b, Astron. Nachr., 253, 195 